\title{
LUCID: The ATLAS Luminosity Detector
}

\author{
Federico Lasagni Manghi, on behalf of the LUCID Collaboration* \\ University and INFN Bologna \\ E-mail: flasagni@cern.ch
}

\begin{abstract}
The LUCID detector is the main luminosity provider of the ATLAS experiment and the only one able to provide a reliable luminosity determination in all beam configurations, luminosity ranges and at bunch-crossing level. LUCID was entirely redesigned in preparation for Run 2: both the detector and the electronics were upgraded in order to cope with the challenging conditions expected at the LHC center of mass energy of $13 \mathrm{TeV}$ and with $25 \mathrm{~ns}$ bunch-spacing. An innovative calibration system based on radioactive $207 \mathrm{Bi}$ sources deposited on the quartz window of the readout photomultipliers was implemented, resulting in the ability to control the detectors long time stability at a percent level. A description of the detector and its readout electronics is given as well as preliminary results of the ATLAS luminosity measurement and related systematic uncertainties.
\end{abstract}

The 39th International Conference on High Energy Physics (ICHEP2018)

4-11 July, 2018

Seoul, Korea

${ }^{*}$ Speaker. 


\section{Introduction: Luminosity and ATLAS}

In High Energy Physics experiments there is a parameter, called luminosity $(L)$ that correlates the interaction rate of any given physical process with its cross-section: $R=L \sigma$. The luminosity can be computed from the parameters of the accelerator as:

$$
L=\frac{f_{L H C} \sum_{i=1}^{n_{b}} N_{i 1} N_{i 2}}{4 \pi \sigma_{x} \sigma_{y}}
$$

where $f_{L H C}$ is the revolution frequency of the accelerator (LHC in this case), $n_{b}$ is the number of colliding bunches, $N$ is the number of protons per bunch in beam 1 and 2 and $\sigma$ represents the beam width in the two coordinates transversal to the beam direction.

In ATLAS [1] the luminosity is measured for three main reasons:

- the LHC control room needs to know the luminosity delivered to ATLAS in order to optimize collisions; this measurement is provided every $\sim 2 \mathrm{~s}$ in real time;

- the ATLAS detector uses the luminosity in the trigger system to calculate the trigger prescalings; these quantities need to be tuned on the base of the average number of interactions per bunch crossing ( $\mu=L \sigma_{\text {inel }}$ ); this measurement is performed over a period called Luminosity Block $(\mathrm{LB})$ that lasts $\Delta t(L B) \sim 60 \mathrm{~s}$;

- finally, the integrated luminosity corresponding to a given dataset is necessary off-line in every cross-section measurement and its uncertainty represents one of the main systematic uncertainties when measuring many cross-sections.

The ATLAS detector has two dedicated luminosity detectors, LUCID and BCM, and several subdetectors that can be used to measure $L$. Generally speaking, the dedicated detectors have the advantage of being independent from the acquisition chain and trigger system, of operating in every $L$ range, of being uncorrelated with the physics measurements and of being able to provide a quick, bunch-by-bunch measurement. Other ATLAS sub-detectors (Inner Tracker, EMEC, FCal, TILE, TPX) provide complementary measurements, having a larger area and more information that can be used off-line to improve the measurement. In 2017, LUCID managed to provide a very stable $L$ measurement, within $1.3 \%$ of the other measurements, after some corrections from track counting, as shown in figure 1.

\section{LUCID design}

Since its conception it was clear that LUCID had to possess a number of features to become the stable and reliable detector it is today:

- Fast and radiation hard particle detection; Cherenkov light in gas (part of Run I) and in quartz PMT windows have been used.

- Simple layout, with a small number of channels, with PMT's pointing in the direction of the interaction point. 


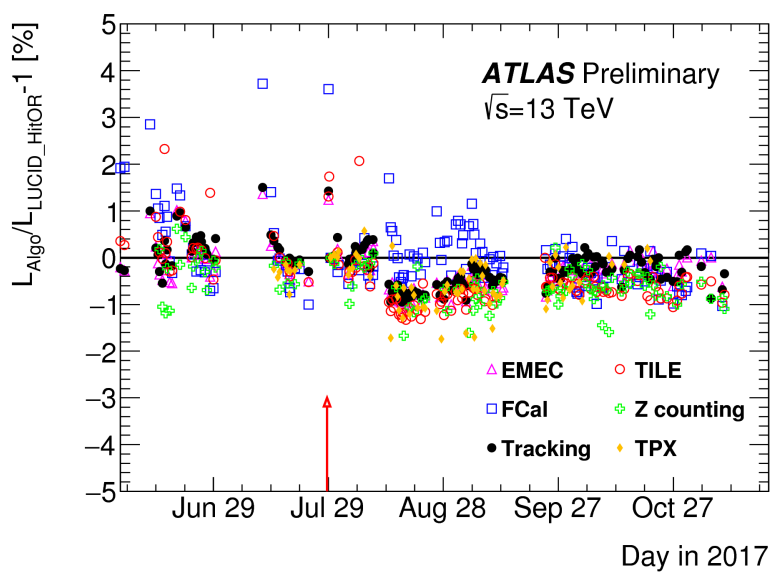

Figure 1: Comparison of the main luminosity detectors/algorithms to the LUCID hit-counting algorithm [2]. The results are mostly within $1.3 \%$ of the LUCID measurements

- Fast electronics, able to operate at the full ATLAS interaction rate, providing hit counting and charge measurement (see section 4).

- Stable measurement, achieved thanks to the PMT gain calibration system.

- Redundancy, obtained by dividing the system into several independent sub-detectors.

- Flexibility, to switch between PMT groups and the possibility to use spare PMT's in case of failures.

The Run 1 LUCID was optimized for Run 1 conditions: low occupancy and 50 ns bunch-spacing. Light was produced both in gas cylinders coupled to the PMT's and in the quartz windows themselves. The gas was shown to suffer from pile-up effects and was removed. The electronic system consisted in signal discriminators placed outside of the experimental caverns, coupled to a digital logic board named LUMAT. As a consequence on the length of the cables, going from the PMT's to the discriminators $(\sim 100 \mathrm{~m})$, the signal was too broad for the upcoming Run 2 bunch spacing of only $25 \mathrm{~ns}$.

During the long shut-down LUCID was updated to cope with the new conditions and to improve on the weaknesses observed during Run 1:

- new, smaller PMT's (Hamamatsu R760) replaced the Run 1 PMT’s (R762);

- two different calibration systems were implemented to monitor the gain variation;

- mu-metal shielding cylinders were used to reduce the impact of stray magnetic fields;

- new dedicated boards were developed to provide early digitization (a few meters from the detector) and discrimination.

LUCID consists of two detector arms, labelled side $A$ and side $C$. For each arm, 4 groups of 4 PMT's are placed around the beam-pipe, pointing towards the interaction point, while 4 quartz fibre bundles are read by PMT's placed in a shielded area. 


\section{PMT's and Fibres}

Monte Carlo simulations performed at the end of Run 1 revealed that the combined effects of increased luminosity and energy and the change from a stainless steel beam-pipe to an aluminium one would produce a $60 \%$ to $80 \%$ increase in PMT rate [3]. A PMT with a smaller window was, thus, chosen for Run 2, going from Hamamatsu R762 (14 mm window diameter) to the similarly built R760 (10 mm window diameter). Each detector arm features 20 PMT's, divided in 5 groups:

- The so-called Bi PMT's compose the main luminosity group;

- The Bi2 PMT's provides the backup measurement and is read-out by a different electronic chain to ensure maximum redundancy;

- The Spare PMT's are inactive, each connected to the same electronic chain as a Bi PMT, in order to replace it in case of failure;

- The BiM group is composed of PMT's modified by depositing a layer of aluminium in a circular corona inside the quartz window, reducing the effective acceptance diameter to $7 \mathrm{~mm}$;

- The Fibers have the PMT's placed in the shielded region and read the light coming from the quartz fibre bundles (the Cherenkov medium); each bundle is composed of 37 fibres.

Describing the characterization of the PMT's before installation goes beyond the scope of this paper. Detailed information can be found in [4].

During the 2015-2016 winter shutdown the Bi2 PMT's were changed together with an improvement of the whole gain calibration system. Due to this, during 2018 operations they have become the main luminosity measurement group.

The PMT High Voltage is provided by a CAEN SY1527 crate that is operated through a Data Control System (DCS) based on WinCCOA 3.15. This system also monitors PMT currents, crate temperatures and calibration data.

\section{Electronics and Data Acquisition}

The electronic system of LUCID in Run 2 consists of two independent acquisition chains, each consisting of two LUCROD boards, installed in crates next to the detectors, and a LUMAT board installed in the electronics cavern to combine the data from the two sides and provide fast luminosity algorithms.

Each LUCROD board has 16 input channels, each connected to a different PMT or other analog input. Its main components are:

- a low noise amplifier and a $480 \mathrm{MHz}$ FADC for each input channel;

- a Xilinx Spartan IV FPGA for each pair of inputs, integrating the input signal over each bunch crossing and discriminating hits;

- an additional FPGA implementing luminosity algorithms and optical link communication; 
- an optical link transceiver to transmit discriminated signals (hits) to the LUMAT board using transceivers that send serialized 16-bit words at $100 \mathrm{MHz}$;

- an analog amplified output for each input;

The board is not radiation hard, but it is located in a shielded area: occasional Single Event Upsets have been observed, and are automatically detected and fixed by firmware reload. The single-PMT hits and charge and single-side algorithms are published directly from the LUCROD's at every Luminosity Block (LB).

The LUMAT motherboard uses 4 different mezzanine cards:

- a main processing mezzanine featuring an Altera Stratix II FPGA, where event-based (at least a PMT above threshold) and hit-based (number of PMT's above threshold) algorithms, with and without coincidence between both sides, are computed;

- two custom mezzanines used for optical communication with the LUCROD's, featuring a Spartan VI FPGA and 4 optical transceivers, receiving data from 2 LUCROD's, on side A and side $\mathrm{C}$, and synchronizing them to the same bunch-crossing ID;

- a TTC mezzanine to get clock information from ATLAS;

an Altera Cyclone FPGA handles VME communication.

Each LUMAT board processes data belonging to two different PMT groups, computing 6 algorithms for each of them. The results of these algorithms are sent to LHC every $2 \mathrm{~s}$, summed over all BCID's, and to ATLAS every LB, BCID per BCID.

The LUCID DAQ system is completed by the DAQ software, integrated in the ATLAS TDAQ infrastructure. It serves three main functions: data publication, performance monitoring and automatic error detection and recovery (when suitable).

\section{Gain Calibration}

During Run 1 a loss of up to 5\% due to the degradation of the PMT gain has been observed after the large current drawn in physics runs, with a particularly large effect for new PMT's. Two different calibration methods were, thus, implemented in the Run 2 LUCID to monitor the gain:

- calibration fibres carrying light from a LED directly to the PMT, method now used only for the Fiber PMT's;

- Bi207 sources deposited on the PMT windows, emitting electrons from internal conversion and producing Cherenkov light, a very effective method now used in every PMT positioned around the beam-pipe.

In both methods the PMT amplitude or charge response to a known signal (LED or bismuth) is measured between physics fills and compared to a reference value. The HV is adjusted accordingly: $\Delta H V=\alpha \times\left(1-R_{A / C}\right)$, where $R$ is the ratio to the reference value and $\alpha \sim 110$ in LUCID. The values are averaged over circa 200000 measurements and the procedure is repeated more than once to be sure to obtain a final stable value. In 2016 the calibration was performed using the average 
signal charge above threshold while, starting from 2017, the PMT's that measure $L$ through hit counting are calibrated measuring the average amplitude. The calibration data is elaborated by the DAQ software and passed to the DCS system, where the high voltage corrections are computed and applied. Figure 2 shows the Bi207 amplitude distribution, compared to data from a physics run, together with the applied HV increase as a function of time over three years.
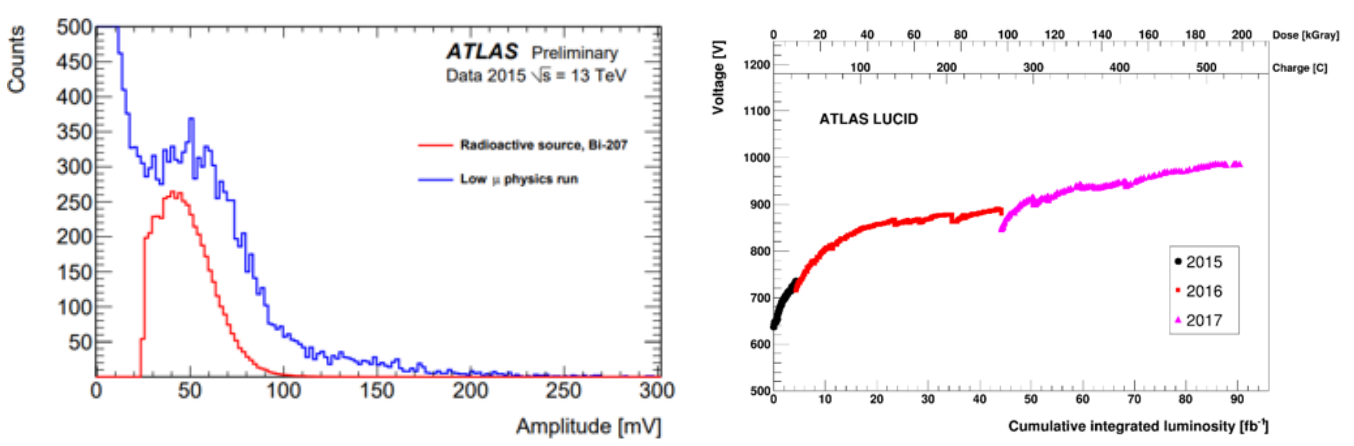

Figure 2: Comparison of the amplitude distribution from Bi source and a low- $\mu$ run (left) [5]. The increase in PMT HV as a function of the integrated luminosity (right) [3].

\section{Absolute luminosity}

The final aim of LUCID is to provide an integrated $L$ measurement independent of the physics data. An absolute calibration is, thus, necessary. This is performed using a technique called Van der Meer Scan: in a dedicated run the beam separations are shifted in steps of $50 \mu \mathrm{m}$ in the horizontal and vertical directions; a visible measured interaction rate $\mu_{v i s}$ is measured together with a convoluted beam width. Together with proton current measurements, the detector is calibrated. Since this scan is performed at low $\mu$ the non linearities of the LUCID hit algorithms with $\mu$ must be evaluated and corrected and a corresponding systematic uncertainty must be assessed. For this, the tracking algorithm of the Inner Detector is used.

\section{Conclusion}

LUCID showed very stable and reliable performance in Run 2 due to its design solutions and its operation during data taking. Thanks to the design solutions adopted, $L$ was measured with an uncertainty of $2.1 \%, 2.2 \%$ and $2.4 \%$ (preliminary) in 2015, 2016 and 2017 respectively.

\section{References}

[1] ATLAS Collaboration, 2008 JINST 3 S08003.

[2] http://atlas.web.cern.ch/Atlas/GROUPS/PHYSICS/PLOTS/LUMI-2017-001

[3] G. Avoni et al., 2018 JINST 13 no.07, P07017.

[4] G. L. Alberghi et al., 2016 JINST 11 no.05, P05014.

[5] https://twiki.cern.ch/twiki/bin/view/AtlasPublic/ForwardDetPublicResults 